\title{
OFDM PAPR reduction for image transmission using improved tone reservation
}

\author{
Zainab Noori Ghanim, Buthaina M. Omran \\ Department of Electronic and Communication, Baghdad University, Iraq
}

\begin{tabular}{l} 
Article Info \\
\hline Article history: \\
Received Dec 30, 2019 \\
Revised Jun 16, 2020 \\
Accepted Aug 10, 2020 \\
\hline
\end{tabular}

\section{Keywords:}

OFDM

PAPR

TR

\begin{abstract}
High peak to average power ration (PAPR) in orthogonal frequency division multiplexing (OFDM) is an important problem, which increase the cost and complexity of high power amplifiers. One of the techniques used to reduce the PAPR in OFDM system is the tone reservation method (TR). In our work we propose a modified tone reservation method to decrease the PAPR with low complexity compared with the conventional TR method by process the high and low amplitudes at the same time. An image of size $128 \times 128$ is used as a source of data that transmitted using OFDM system. The proposed method decrease the PAPR by $2 \mathrm{~dB}$ compared with conventional method with keeping the performance unchanged. The performance of the proposed method is tested with several numbers of subcarriers; we found that the PAPR is reduced as the number of subcarriers decreased.
\end{abstract}

This is an open access article under the CC BY-SA license.

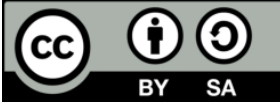

\section{Corresponding Author:}

Zainab Noori Ghanim,

Department of Electronic and Communication Engineering,

Baghdad University,

Baghdad, Iraq.

Email: zainabnoori70@yahoo.com

\section{INTRODUCTION}

Orthogonal frequency division multiplexing (OFDM) is a technique used to transmit a high speed data. The concept of OFDM is the spreading of the high speed transmitted data over a large number of subcarriers [1]. The OFDM system covers the requirements for wireless image and video communication services. The image has big size and needs long time to transmit over wireless channel in OFDM system, the transmitted image is needed to be coded by two types of coding, the first is the source code to reduce redundant data in the image, decreasing the image size and transmission time, the second is the channel code, which is helpful for error detection and correction [2-4].

The peak to average power ratio (PAPR) provides a measure for the variation range of signal power. The PAPR is high in OFDM because the nature of modulation, where multiple subcarriers are added together to form the transmitted signal. These large peaks increase the probability of leakage energy to the neighbor channel, which lead to signal distortion in the output of power amplifier and degrade the error rate performance due to the system constraint to a limited peak power of dynamic range in transmitter amplifiers. Thus, a large power amplifier has needed. However, increasing the large power in amplifier not only reduces the power efficiency but also increase the amplifier cost significantly $[5,6]$.

Several approaches have been introduced to reduce PAPR, but, these methods increase the expense of transmitted signal power, degrade the bit error rate, increase the computational complexity, and lead to loss of data rate, so, a system trade-off is predicted [7]. One can divide the methods used to reduce the PAPR of an OFDM signal to four categories: i) signal distortion, ii) coding methods, iii) probabilistic (scrambling) techniques and iv) pre-distortion methods $[8,9]$. The pre-distortion methods attempt to reorient or spread 
the energy of data symbol. The pre-distortion schemes include the DFT spreading, pulse shaping or pre-coding and constellation shaping. The tone reservation (TR) is one example of constellation shaping methods [10].

Tone Reservation technique has based on reserving some tones to reduce PAPR. The PAPR reduction amount depends on the number of the reserved tones and their position. The performance of TR also depends on the amount of complexity and reserved tones power. This technique can be applied to every type of OFDM system. In this method, transmission of side information is not necessary at the receiver [11]. In this paper an image is used as a source of data, good quality received image with PAPR reduction is the aim of this paper. TR method is used for PAPR reduction, new proposal is introduced to improve the clipping based TR method, by clipping the high amplitudes and amplifying the low amplitudes, the method aims to decrease the number of iterations with parameters changeable at each iteration.

Many papers work on TR method improvement, where Horvath and Botlik made optimization for TR by changing clipping ratio in each iteration to make limits for the peak values. In our work, we make clipping for the maximum values and amplification for minimum values, the PAPR reduction in this work increases with increasing the number of iterations but in our work the increasing of number of iteration is not useful [12]. Ali et al. decrease the complexity of TR technique. The PAPR reduction in this work is less than that in our work [13]. Antonov et al. decrease TR complexity, by randomly generation of different combinations of manipulation symbols, which lead to the additional subcarriers, equally spaced over the signal bandwidth. They need one hundred iterations in their work, but in our work, we need three iterations [14]. Abdelali et al. make combination of TR method and clipping method to improve PAPR reduction. They need high number of iterations and the bit error rate is more than that in our work [15]. Shixian Lu et al. suggest a sliding window, tone reservation PAPR reduction technique. They need fifty iterations to reach to good results but we need three iterations [16]. The modification of Tone Reservation method to reduce PAPR ratio with multicarrier non-orthogonal signal is introduced in [17].

The organization of this paper is as follows: an overview of OFDM system and PAPR definition has introduced in section 2. In section 3 an explanation for tone reservation method will be presented. Section 4 presents the proposed algorithm. Performance measurements in section 5. In section 6 the simulation results for the used technique will be presented and discussed. Finally, the conclusions for the used technique has shown in section 7 .

\section{OFDM SIGNAL MODEL AND PEAK TO AVERAGE POWER RATIO (PAPR)}

An OFDM systems use orthogonal and independent subcarriers, where the modulation process can be easily implemented using simple N-point inverse fast Fourier transform (IFFT) operation, the output signal is a summation of modulated complex harmonics [12] that can be expressed as [13, 18]:

$$
x(t)=\frac{1}{N} \sum_{k=0}^{N-1} s_{k} e^{j 2 \pi f_{k} t} \quad 0 \leq t \leq T_{S}
$$

where $s_{k}$ is the data symbol transmitted on the $k^{\text {th }}$ sub carrier $f_{k}$, the subcarrier index is denoted by $\mathrm{k}$ and $T_{s}$ is the symbol duration.

OFDM systems introduce high PAPR due to the use of a large number of subcarriers [19], for a continuous time baseband OFDM signal, the definition of PAPR of any signal is the proportion of the maximum instantaneous power of the signal to its mean power. If $x(t)$ represents a baseband transmitted OFDM signal, then

$$
\operatorname{PAPR}[x(t)]=\frac{\underset{0 \leq t \leq T_{S}}{M A X}|x(t)|^{2}}{P_{a v}}
$$

where, $P_{a v}$ is the average power of $x(t)$ which can be computed in frequency domain because IFFT is a unitary transformation. $T_{s}$ represents the useful duration of an OFDM symbol [8].

The complementary cumulative distribution function (CCDF) has used to describe a distribution of a random variable [11]. The distribution of PAPR may be expressed in terms of (CCDF), which is represents the probability that the PAPR of an OFDM symbol exceeds a given threshold PAPR0, which is denoted as $[1,20]$.

$$
C C D F(P A P R 0)=P(P A P R>P A P R 0)=1-P(P A P R 0)
$$

The CCDF has also used to evaluate the performance of PAPR reduction in OFDM systems [1, 20]. Tone reservation method is one of the many techniques, which have introduced in the literatures to reduce the PAPR of an OFDM signal. 


\section{TONE RESERVATION METHOD}

In TR schemes, some of the total number of tones $N$ are reserved as a peak reserved tones (PRTs) and stored in the reserved tones vector $P R T=\left\{p_{0}, p_{1}, \ldots, p_{Z-1}\right\}$, where $z$ is the size of the PRT set. With TR methods, the OFDM $k$ th data block $\boldsymbol{X}_{\boldsymbol{k}}^{\boldsymbol{T R}}$ configured from two parts, the peak reserved tones that are stored in the PRTs and denoted by $X_{k}^{t r}$ and the unreserved tones $X_{k}$, i.e., [13, 21].

$$
X_{k}^{T R}=X_{k}+X_{k}^{t r} \quad k=0,1,2, \ldots, N-1
$$

There are different methods for tone reservation technique, kernel based and clipping based TR methods have explained below.

\subsection{Kernel based (TR-K) method}

This method creates a reference kernel vector $p_{n}$, which is an impulse function has FFT size, in the kernel vector the reserved tones positions will set to one, and all other values are updated to zero. The kernel will update with a single peak and all the other samples would be zero, but this is impractical because of the limited bandwidth. In every iteration $i$ the maximum amplitude $A^{i}$ of OFDM signal $x^{i}$ and its position $m^{i}$ has to be found. The position of the maximal amplitude of the kernel vector is circularly shifting to the position $m^{i}$, the adding of kernel vector to the original input reduces the peak to a previously determined wanted clipping level. The following equations will explain the method:

$$
\begin{aligned}
& x^{i+1}=x^{i}-\alpha^{i} p_{n}\left(m^{i}\right) \\
& \alpha^{i}=\frac{x^{i}\left(m^{i}\right)}{A^{i}}\left(A^{i}-A_{\max }\right)
\end{aligned}
$$

where $A_{\max }$ is the clipping amplitude and $p_{n}\left(m^{i}\right)$ is the circularly shifted kernel, then the PAPR value is calculated $[12,22]$.

\subsection{Clipping based (TR-C) method}

This method is shown in Figure 1. In clipping method, a generated signal $r_{n}$ is subtracted from the original OFDM symbol $x_{n}$ :

$$
b_{n}^{i}=x_{n}^{i}-r_{n}^{i}
$$

where

$$
r_{n}= \begin{cases}x_{n}, & \left|x_{n}\right|<\operatorname{Amax} \\ \operatorname{Amax} e^{j \emptyset\left(x_{n}\right)}, & \left|x_{n}\right| \geq \operatorname{Amax}\end{cases}
$$

$r_{n}$ is the clipped signal and the maximum clipped signal is $A_{\max }, \emptyset\left(x_{n}\right)$ is the phase of $x_{n}$. In the frequency domain:

$$
\begin{aligned}
& B_{k}=F F T\left(b_{n}\right) \\
& {B^{\prime}{ }_{k}}^{\prime}= \begin{cases}B_{k}, & k \in P R T \\
0, & k \notin P R T\end{cases}
\end{aligned}
$$

where $P R T$ is the matrix of the positions of clipped signals $\left(b_{n} \neq 0\right) . B^{\prime}{ }_{k}$ is the same as $B_{k}$ matrix in the clipped signal position and will be zero in the other positions.

$$
\begin{aligned}
& {b^{\prime}}_{n}=\operatorname{IFFT}\left(B^{\prime}{ }_{k}\right) \\
& x_{n}^{i+1}=x_{n}^{i}+\mu{b^{\prime}}_{n}^{i}
\end{aligned}
$$

The clipped signal $b_{n}^{\prime}$ is added to the original OFDM signal $x_{n}$ with weight parameter $\mu$ and this operation is repeated for several iterations until minimum PAPR is obtained for the resulting signal $y_{n}$, the symbol $i$ denotes for each iteration $[12,22]$. 


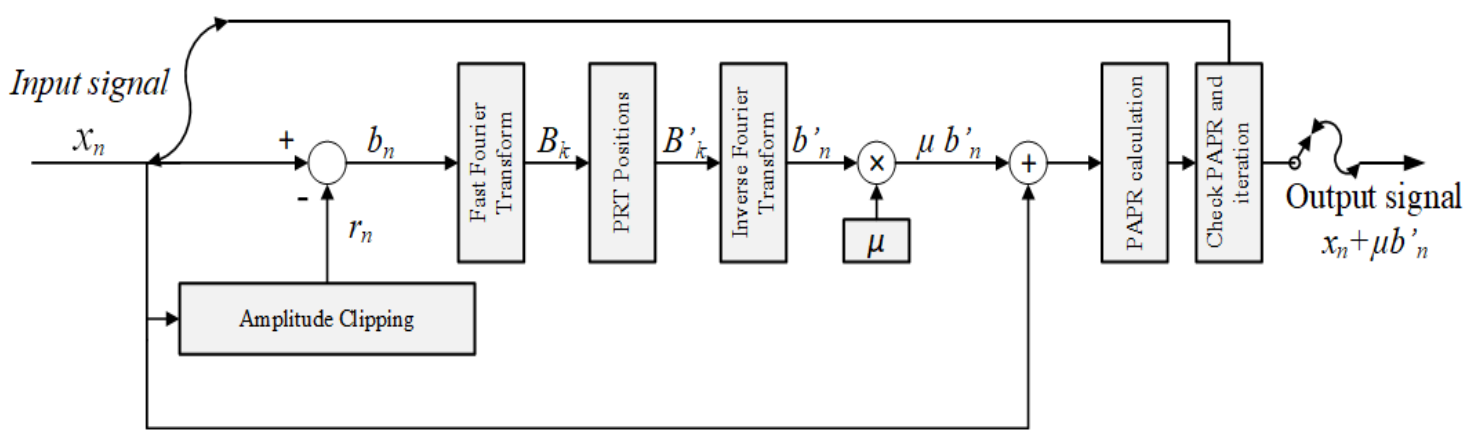

Figure 1. Block diagram of TR-C original method

\section{PROPOSED ALGORITHM}

The proposed work does not need any processing at the receiver as the original TR, but we improve the TR_C method by clipping the maximum peaks of original signal which exceed a limited $A_{\max }$ level and amplify the lowest values of original signal which are lower than a limited level $A_{\min }$, see Figure 2.

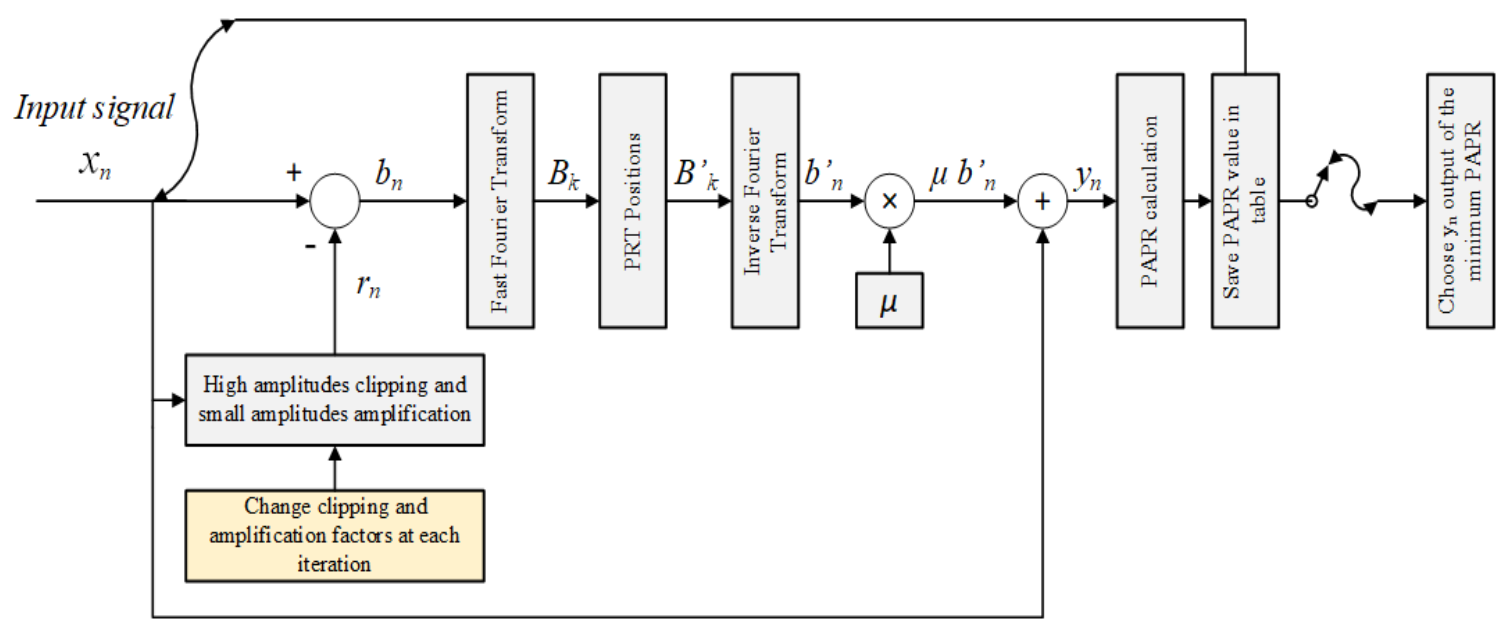

Figure 2. Block diagram of proposed TR-C

$$
r_{n}=\left\{\begin{array}{lr}
x_{n}, & \text { if } A \min <x_{n}<A \max \\
\propto x_{n}, & \text { if } x_{n}<A \min \\
\beta x_{n}, & \text { if } x_{n}>A \max
\end{array}\right.
$$

Where $\alpha$ is a factor more than one multiplied with the original signal to amplify the low levels, $\beta$ is a factor less than 1 multiplied with the original signal to minimize the high levels of signal, by this method the maximum is minimized with keeping the average value of the overall messages close to the original value and minimizing PAPR, see (2). In this technique $A_{\max }$ and $A_{\min }$ are changed in each iteration, their values are ratio of the largest signal $x_{n}$, which is called $x_{\text {nmax }}$. The values of $\alpha$ and $\beta$ are also changed in each iteration. Four cases can be obtained by changing $A_{\max }, A_{\min }, \alpha$ and $\beta$ :

1) Case1: decreasing $\alpha$ by $(1+0.1 M i-0.1 i)$ with increasing $A_{\min }$ by $\left((0.1 i) x_{n \max }\right)$, in the other side decreasing $\beta$ by $(1-0.1 i)$ with increasing $A_{\max }$ by $\left((0.5+0.1 i) x_{n \max }\right)$.

2) Case2: increasing $\alpha$ by $(1+0.1 i)$ with decreasing $A_{\min }$ by $\left((0.5-0.1 i) x_{\text {nmax }}\right)$, in the other side decreasing $\beta$ by $(1-0.1 i)$ with increasing $A_{\max }$ by $\left((0.5+0.1 i) x_{\text {nmax }}\right)$.

3) Case3: decreasing $\alpha$ by $(1+0.1 M i-0.1 i)$ with increasing $A_{\min }$ by $\left((0.1 i) x_{\text {nmax }}\right)$, in the other side increasing $\beta$ by $(0.5+0.1 i)$ with decreasing $A_{\max }$ by $\left((1-0.1 i) x_{\text {nax }}\right)$.

4) Case4: increasing $\alpha$ by $(1+0.1 i)$ with decreasing $A_{\min }$ by $\left((0.5-0.1 i) x_{\text {nmax }}\right)$, in the other side increasing $\beta$ by $(0.5+0.1 i)$ with decreasing $A_{\max }$ by $\left((1-0.1 i) x_{\text {nmax }}\right)$. 
Where the maximum number of iterations is $M i$ and $i$ is the iteration number $(i=1,2, \ldots, M i)$. At each iteration the output $y_{n}$ is obtained as in (14), it is saved in table with its PAPR value, then the output with minimum value of PAPR is taken in this technique.

$$
y_{n}^{i}=x_{n}^{i}+\mu b_{n}^{\prime i}
$$

\section{PERFORMANCE MEASUREMENTS}

The transmitted data in this paper is image. Measurements for checking the received image quality has often used, peak signal to noise ratio (PSNR) and mean squared error (MSE) of the decoded received image are measured with respect to the transmitted one as follows:

$$
\operatorname{MSE}=\frac{1}{\mathrm{MN}} \sum_{\mathrm{i}=1}^{\mathrm{M}} \sum_{\mathrm{j}=1}^{\mathrm{N}}\left|\mathrm{X}_{1}(\mathrm{i}, \mathrm{j})-\mathrm{X}_{2}(\mathrm{i}, \mathrm{j})\right|^{2}
$$

where $\mathrm{M}$ and $\mathrm{N}$ are the image vertical and horizontal dimensions, $X_{1}$ and $X_{2}$ are the transmitted and received images respectively, MSE is a metric used to measure the amount of errors in the received image with respect to the transmitted one. PSNR measures the precision of image decoding, better image quality PSNR is greater than $20 \mathrm{~dB}$ [23-25].

$$
\operatorname{PSNR}=10 \log _{10}\left(\frac{255^{2}}{\mathrm{MSE}}\right)
$$

\section{SIMULATION AND RESULTS}

In this work colored image of Lenna with size $128 \times 128$ is used, the image is coded using Huffman source code and BCH channel code, and transmitted over OFDM system with parameters shown in Table 1, under additive white Gaussian noise (AWGN) channel. Mat-lab 2018a is used in the simulation.

Table 1. Parameters used in simulation

\begin{tabular}{cc}
\hline Name of Parameters & Value \\
\hline System & OFDM \\
Modulation & 16QAM \\
Num. of subcarriers & 64 to 1024 \\
Band width & $20 \mathrm{MHz}$ \\
Num. of symbols & 49152 \\
Value of $\mu$ & -1 \\
\hline
\end{tabular}

CCDF is computed for the transmitted OFDM signal without any PAPR reduction and is compared with CCDF of OFDM signal with conventional TR technique and the CCDF of signal with the four cases of proposed TR technique (FFT size=256), see Figure 3, from this figure the PAPR is reduced about $2 \mathrm{~dB}$ for proposed TR with respect to the PAPR of FFT signal without any PAPR reduction, the PAPR is similar among the four cases of proposed TR, it is clear that the proposed TR PAPR with $M i=3$ reduce the PAPR more than for conventional TR with $M i=10$.

In the other side the CCDF and bit error are computed and compared for the signal with different subcarriers number, the first case of proposed TR is computing with $\mathrm{Mi}=3$. The CCDF of the signal with different number of subcarriers is shown in Figure 4, from this figure we find that the PAPR reduced as the number of sub carriers increased. The bit error for them is shown in Figure 5, from this figure we find that the larger number of subcarriers has the less BER at the same signal to noise ratio (SNR).

The CCDF of the OFDM signal with proposed TR first case and FFT size $=256$ are shown and compared in Figure 6 for two different maximum number of iterations, for $\mathrm{Mi}=3$ and $\mathrm{Mi}=5$, from the figure we can see that the PAPR reduction with $\mathrm{Mi}=5$ is more than the PAPR reduction with $\mathrm{Mi}=3$ by a small value, at the same time the $\mathrm{BER}=1.0173 \mathrm{e}-05$ at $\mathrm{SNR}=10 \mathrm{~dB}$ for $\mathrm{Mi}=3$, but the $\mathrm{BER}=3.0518 \mathrm{e}-05$ at $\mathrm{SNR}=10 \mathrm{~dB}$ for $\mathrm{Mi}=5$, the $\mathrm{BER}$ for $\mathrm{Mi}=5$ is worse than that for $\mathrm{Mi}=3$ by a small value.

The proposed TR (first case and $\mathrm{Mi}=3$ ) is used to reduce the PAPR for Lenna image which is transmitted with OFDM. The original and the received image are shown in Figure 7 for different numbers of subcarriers with $\mathrm{SNR}=10 \mathrm{~dB}$. The received image with FFT size=256, but for different values of SNR are shown in Figure 8. From Figure 7, we found that image transmitted with higher number of sub carriers has the better peak signal to noise ratio (PSNR), and from Figure 8 we show that the image at smallest SNR has the smallest PSNR. 


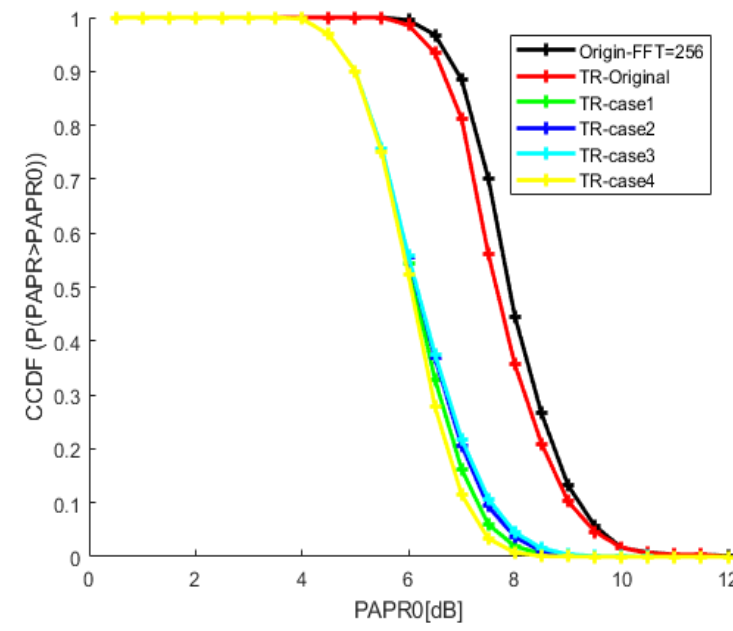

Figure 3. CCDF of PAPR for OFDM signal with four cases of proposed TR PAPR reduction, original OFDM signal without PAPR reduction and with original TR-C method

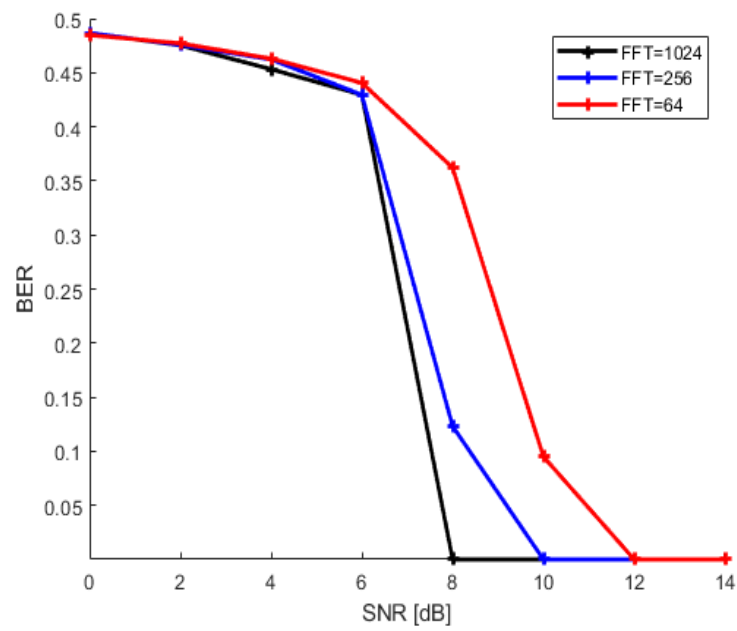

Figure 5. BER of the OFDM signal with proposed TR for different number of carriers

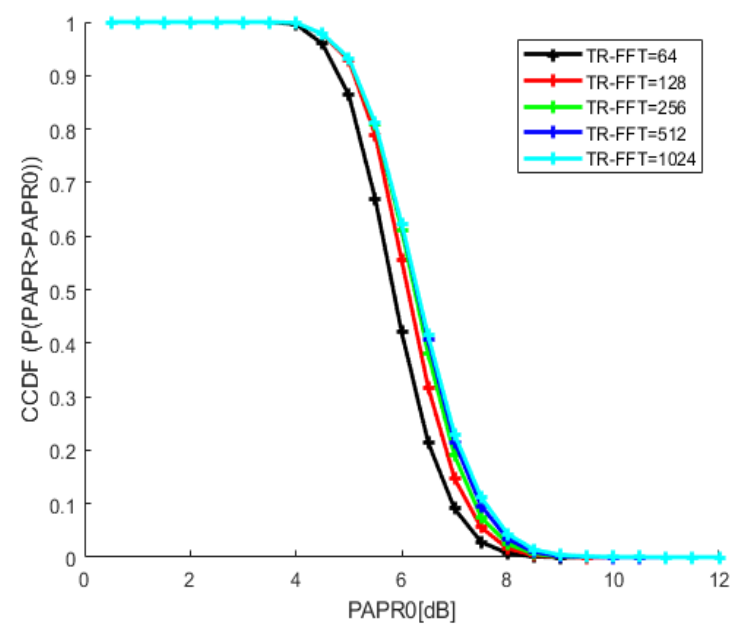

Figure 4. CCDF of PAPR for OFDM signal with proposed TR first case and different number of subcarriers

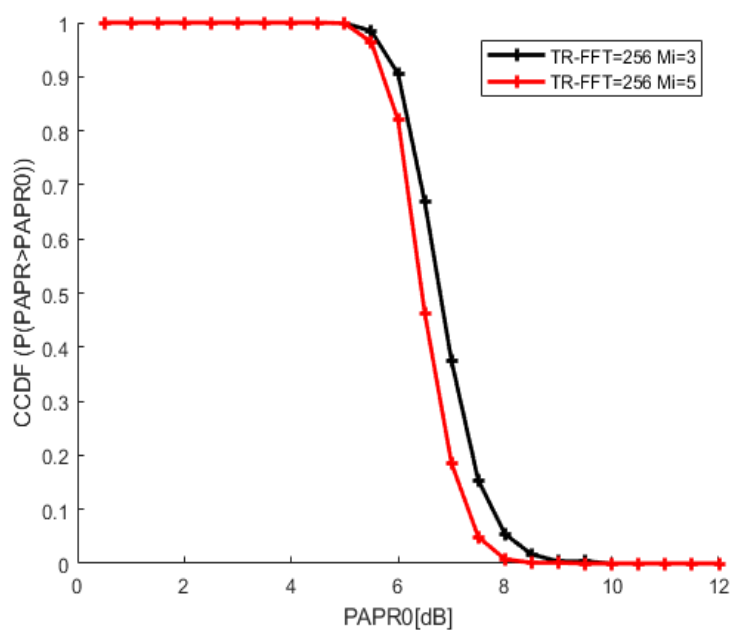

Figure 6. CCDF of PAPR for OFDM signal with proposed TR for two different maximum number of iterations

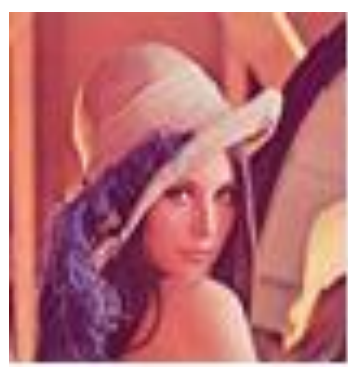

(a)

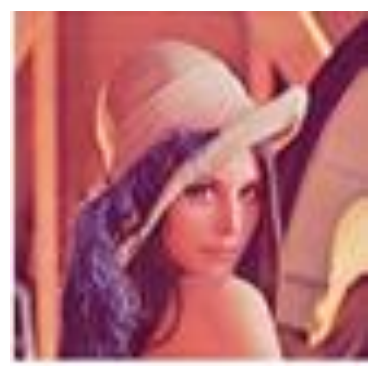

(b)

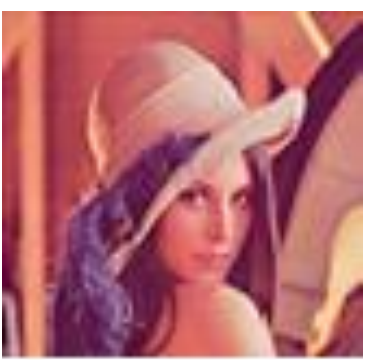

(c)

Figure 7. Lenna image: original and received image with different FFT size, (a) Original image, (b) $\mathrm{SNR}=10 \mathrm{~dB}, \mathrm{FFT}$ size $=64, \mathrm{PSNR}=46$, (c) $\mathrm{SNR}=10 \mathrm{~dB}$, FFT size $=128$. PSNR=71.5 


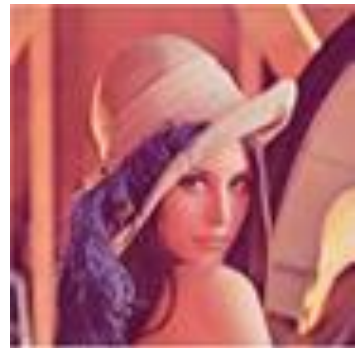

(a)

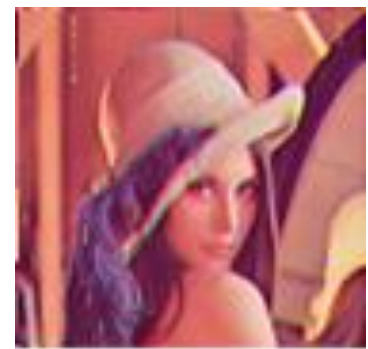

(b)

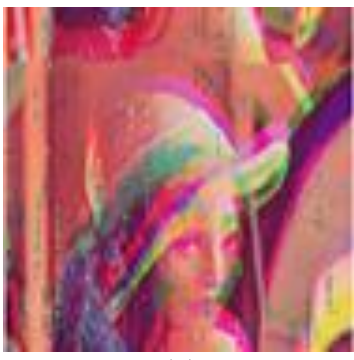

(c)

Figure 8. The received Lenna image with different SNR, (a) SNR=10 dB, FFT size=256, PSNR=71.5,

(b) $\mathrm{SNR}=8 \mathrm{~dB}, \mathrm{FFT}$ size $=256, \mathrm{PSNR}=20.7147$, (c) $\mathrm{SNR}=6 \mathrm{~dB}$, FFT size $=256$, $\mathrm{PSNR}=13.5189$

\section{CONCLUSION}

From the simulation results, one can concludes that clipping the high amplitudes and amplifying the low amplitudes lead to reduce the number of iterations compared with the conventional TR method and enhance the overall system performance. The proposed method is applied in this paper on the image transmission; this method can also applied on another data such as speech or video transmission.

\section{REFERENCES}

[1] H. Ait-Saadi, et al., "A PAPR Reduction for OFDM Signals Based on Self-Adaptive Multipopulation DE algorithm," International Journal of Electrical and Computer Engineering (IJECE), vol. 7, no. 5, pp. 2651-2660, Oct 2017.

[2] M. Chandra, et al., "Image Transmission through Wireless Channel: A review," IEEE 1st International Conference on Power Electronics, Intelligent Control and Energy Systems, Delhi, India, pp. 1-4, 2016.

[3] N. F. Soliman, et al., "Robust Image Transmission with OFDM over an AWGN Channel," 28th National Radio Science Conference, Egypt, pp. 1-8, 2011.

[4] T. Wenisch, et al., "Combined Error Correcting and Compressing Codes," IEEE International Symposium on Information Theory, Washington DC, USA, 2001.

[5] M. D. Rozaini, et al., "Median codeword shift (MCS) technique for PAPR reduction with low complexity in OFDM system," International Journal of Electrical and Computer Engineering (IJECE), vol. 9, no. 6, pp. 4882-4888, 2019.

[6] M. I. Youssef, et al., "Image multiplexing using residue number system coding over MIMO-OFDM communication system," International Journal of Electrical and Computer Engineering (IJECE), vol. 9, no. 6, pp. 4815-4825, 2019.

[7] M. M. Mowla and S. M. M. Hasan, "Performance Improvement of PAPR Reduction for OFDM Signal in LTE System," International Journal of Wireless \& Mobile Networks, vol. 5, no. 4, pp. 35-47, 2013.

[8] Y. S. Cho, et al., "MIMO OFDM wireless communications with Matlab," John Wiley \& Sons, 2010.

[9] C. Choudhary and V. Gupta, "A Study of Performance Enhancement Schemes for Multicarrier Transmission," International Journal of Computer Applications, vol. 68, no. 5, pp. 50-54, 2013.

[10] K. K. Sen and V. sahu, "PAPR Reduction Using PTS with DCT SLM Technique," International Journal of Computer Network and Security, vol. 1, pp. 27-29, 2014.

[11] K. Mhatre and U. P. Khot, "Efficient Selective Mapping PAPR Reduction Technique," Procedia computer science, vol. 45, pp. 620-527, 2015.

[12] B. Horvath and B. Botlik, "Optimization of Tone Reservation-Based PAPR Reduction for OFDM Systems," RADIOENGINEERING, vol. 26, no. 3, pp. 791-797, 2017.

[13] M. Ali, et al., "PAPR and BER Performances of OFDM System with Novel Tone Reservation Technique Over Frequency Non-Selective Fading Channel," Advances in Science, Technology and Engineering Systems Journal, vol. 4, no. 1, pp. 225-230, 2019.

[14] E. O. Antonov, et al., "Reduced Complexity Tone Reservation Peak-to-Average Power Ratio Reduction Algorithm for SEFDM Signals," 39 International Conference on Telecommunications and Signal Processing (TSP), Vienna, Austria, pp. 445-448, 2016.

[15] H. Abdelali, et al., "New Technique Combining the Tone Reservation Method with Clipping Technique to Reduce the Peak-to-Average Power Ratio," International Journal of Electrical and Computer Engineering (IJECE), vol. 8, no. 6 , pp. 5215-5226, 2018.

[16] S. Lu, et al., "Sliding Window Tone Reservation Technique for the Peak-to-Average Power Ratio Reduction of FBMC-OQAM Signals," IEEE Wireless Communication Letters, vol. 1, no. 4, pp. 268-271, 2012.

[17] D. C. Nguyen, et al., "The Effectiveness of Tone Reservation Method for Peak-to-Average Power Ratio Reduction of SEFDM Signals with Optimal Envelopes," 2019 IEEE International Conference on Electrical Engineering and Photonics, pp. 165-168, 2019. 
[18] A. Lahcen, et al., "Low Computation Complexity PTS Scheme for PAPR Reduction of MIMO-OFDM Systems," $10^{\text {th }}$ International Conference interdisciplinarity in engineering (INTER-ENG 2016), vol. 181, pp. 876-883, 2017.

[19] P. P. Ann and R. Jose, "Comparison of PAPR Reduction Techniques in OFDM systems," 2016 International Conference on Communication and Electronics Systems (ICCES), pp. 1-5, 2016.

[20] M. H. Aghdam and A. A. Sharifi, "PAPR reduction in OFDM systems: An efficient PTS approach based on particle swarm optimization," ICT Express, vol. 5, pp. 178-181, 2019.

[21] S. Vangala and A. Sundru, "Overlapped Scaling Tone Reservation method for PAPR Reduction in OFDM/OQAM Systems," 6th International Conference On Advances In Computing \& Communications, ICACC 2016, Cochin, India, Procedia computer science, vol. 93, pp. 632-638, 2016.

[22] M. Mrou'e, et al., "Performance and Impleme.ntation Evaluation of TR PAPR ReductionMethods for DVB-T2," International Journal of Digital Multimedia Broadcasting, vol. 2010, pp. 1-10, 2010.

[23] I. Develia and Y. Kabalci, "Proposal of an experimental data and image transmission system and itspossible application for remote monitoring smart grids," Journal of Applied Research and Technology, vol. 15, pp. 303-310, 2017.

[24] Uzma, et al., "A MIMO-OFDM Based Secure and Robust Communication System for IOT Driven Healthcare," International Journal of Recent Technology and Engineering (IJRTE), vol. 7, no. 6, pp. 1208-1221, 2019.

[25] R. Muthiya, et al., "Pseudorandom Noise Sequence of Digital Watermarking Algorithim based on Discrete Wavelete Transform Using Medical Image," The International Arab Journal of Information Technology, vol. 15, no. 4, pp. 769-776, 2018.

\section{BIOGRAPHIES OF AUTHORS}

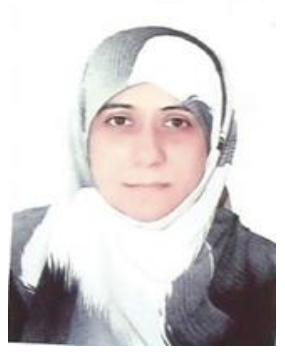

Zainab Noori Ghanim was born in Baghdad, Iraq, on July 16, 1970. She received the B.Sc. and M.Sc. degrees in electronics and communication engineering from the University of Baghdad in 1992 and 1999, respectively. From 1992 to 1999 she worked as an engineer in electronics and communication engineering department in the University of Baghdad. From 1999 to 2011 she worked as an assistant lecturer and from 2011 she worked as lecturer in electronics and communication engineering department in the University of Baghdad.

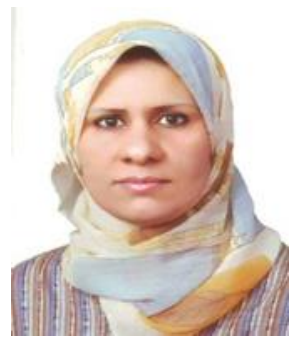

Buthaina Mosa Omran was born in Baghdad, Iraq, on October 12, 1974. She received the B.Sc. and Ph.D. in electronics and communication engineering from University of Baghdad in 1996 and 2007, respectively, and received the M.Sc. degree in Modern communication from the Nahrain University in 2000. She worked in the field of Satellite Communication in Al-Battani Company/Ministry of Sciences and Technology from (2001-2003), and worked in the field of Optical Communications in Al-Razi Company/ Ministry of Industry from (2001-2003). Since 2000 she works as lecturer in electronics and communication engineering department in the university of Bahghdad. From 2018 till now, she is the head of the electronics and communication engineering department in the university of Baghdad. 